\title{
Two-dimensional, phenanthroline-based, extended $\pi$-conjugated molecules for single-molecule conduction
}

\author{
Sören Wohlthat ${ }^{1}$, Fabian Pauly ${ }^{2,3}$ and Jeffrey R Reimers ${ }^{1,4}$ \\ ${ }^{1}$ School of Chemistry, The University of Sydney, Sydney, NSW 2006, Australia \\ ${ }^{2}$ Institut für Theoretische Festkörperphysik and DFG-Center for Functional Nanostructures, \\ Universität Karlsruhe, 76128 Karlsruhe, Germany \\ ${ }^{3}$ Institut für Nanotechnologie, Forschungszentrum Karlsruhe, 76021 Karlsruhe, Germany \\ E-mail: reimers@chem.usyd.edu.au
}

\begin{abstract}
The conduction properties of phenanthroline-terminated, polycyclic extended $\pi$-conjugated molecular wires are investigated using density functional theory (DFT) in combination with Green's function techniques and group theory. While these molecules could possibly be thought of as accessible graphene-like fragments, they are calculated to conduct poorly. The decay constant for their exponential decrease of conductance with length is in excess of $0.6 \AA^{-1}$ for the addition of internal fused quinoxaline groups and in excess of $0.9 \AA^{-1}$ for the addition of internal pyrazine-fused pyrene groups. Furthermore, while the bidentate phenanthroline connectors adhere strongly to gold, they are sometimes predicted to be less conductive than related monodentate connectors. Careful design is thus required for any graphene-like extended $\pi$-system intended for single-molecule conduction applications.
\end{abstract}

The anchoring of organic molecules to metals is an important issue in molecular electronics [1]. Phenanthroline has been suggested as such an 'alligator clip' for attaching molecules with extended $\pi$-conjugation to surfaces [2]. It is particularly applicable for linking oligoporphyrins, molecules with useful conduction properties [2]. Such systems can be thought of as small synthetically feasible models for graphene-like nanoribbons, materials also of great interest for molecular electronics applications [3-6]. Studies of phenanthrolinebased molecules include electrochemical and spectroscopic properties [7] as well as their usefulness for facilitating intramolecular electron transfer processes involving chelated metals [2]. Here, we investigate their single-molecule conduction by means of density functional theory (DFT) in combination with Green's function techniques. We look especially at the length dependence of their conductance as well as the effectiveness of the bridging group in several chemical configurations.

4 Author to whom any correspondence should be addressed.
The investigation of these azine-linked, extended $\pi$ conjugated molecules is intrinsically linked with the general DFT problem of band line up [8]. Owing to the asymptotic potential error inherent in most density functionals, the highest-occupied molecular orbital (HOMO) of the organic molecule is poorly represented in relation to the Fermi energy of metals, and this significantly affects hole conductivity. In addition, the large disparity between the DFT band gap (orbital energy difference between the HOMO and the lowest-unoccupied molecular orbital (LUMO)) and the corresponding electrochemical potential difference affects the perceived electron conductivity $[8,9]$. In order to overcome these problems, we apply empirical corrections to the DFT calculations in the spirit of Quek et al [10] and Mowbray et al [11].

We explore the properties of the phenanthroline-based, extended $\pi$-conjugated molecules shown chemisorbed to gold electrodes in figure 1: tetrapyrido[3,2- $a ; 2^{\prime}, 3^{\prime}-c ; 3^{\prime \prime}, 2^{\prime \prime}$ $\left.h ; 2^{\prime \prime \prime}, 3^{\prime \prime \prime}-j\right]$ phenazine (TPPHZ), dipyrido $\left[3^{\prime}, 2^{\prime}: 5,6 ; 2^{\prime \prime}, 3^{\prime \prime}: 7,8\right]$ 
Table 1. The binding energy, the deduced dominant conduction channels, the most significant molecular orbitals, and the conductance both before and after the scissor correction to the orbital energies, for the molecules and gold ligation patterns considered.

\begin{tabular}{|c|c|c|c|c|c|c|c|}
\hline Molecule & Structure & $\begin{array}{l}\mathrm{Au}-\mathrm{N} \text { bond } \\
\text { length }(\AA)\end{array}$ & $\begin{array}{l}\text { Binding } \\
\text { energy (eV) }\end{array}$ & $\begin{array}{l}\text { Dominant } \\
\text { conduction } \\
\text { channels }\end{array}$ & $\begin{array}{l}\text { Dominant } \\
\text { molecular } \\
\text { orbitals }\end{array}$ & Conduct & Corrected \\
\hline 1,4-benzenediamine & Linear & 2.5 & 0.86 & $\mathrm{~A}^{\prime}$ & $a^{\prime}(\pi)$ НОМО & 0.086 & $0.013^{\mathrm{a}}$ \\
\hline 1,4-benzenediamine & Diagonal & 2.1 & 0.58 & $\mathrm{~A}^{\prime}$ & $a^{\prime}(\pi)$ HOMO & 0.027 & $0.012^{\mathrm{a}}$ \\
\hline BQPY & Bidentate & 2.6 & 2.30 & $3 \times A$ & $\begin{array}{l}b_{1}(\pi), a_{1}(\sigma) \\
b_{2}(\sigma) \text { HOMOs }\end{array}$ & $1.7 \times 10^{-8}$ & $6.4 \times 10^{-9}$ \\
\hline TPPHZ & Bidentate & 2.3 & 2.16 & $\mathrm{~A}^{\prime \prime}$ & $b_{1}(\pi)$ LUMO & $1.2 \times 10^{-4}$ & $1.0 \times 10^{-5}$ \\
\hline TATPP & Bidentate & 2.4 & 2.14 & $\mathrm{~A}^{\prime \prime}$ & $b_{1}(\pi)$ LUMO & $2.3 \times 10^{-6}$ & $6.7 \times 10^{-7}$ \\
\hline TATPP-1-linear & Monodentate & 2.4 & 1.16 & $A^{\prime \prime}$ & $b_{1}(\pi)$ LUMO & $6.4 \times 10^{-5}$ & $2.8 \times 10^{-7}$ \\
\hline TATPP-1-diagonal & Monodentate & 2.4 & 1.17 & $A^{\prime \prime}$ & $b_{1}(\pi)$ LUMO & $2.7 \times 10^{-5}$ & $1.6 \times 10^{-6}$ \\
\hline TATPP-2 & Mixed & 2.4 & 1.69 & $\mathrm{~A}^{\prime \prime}$ & $b_{1}(\pi)$ LUMO & $9.9 \times 10^{-6}$ & $7.0 \times 10^{-7}$ \\
\hline
\end{tabular}

${ }^{a}$ Experiment $0.0064 G_{0}[17]$.

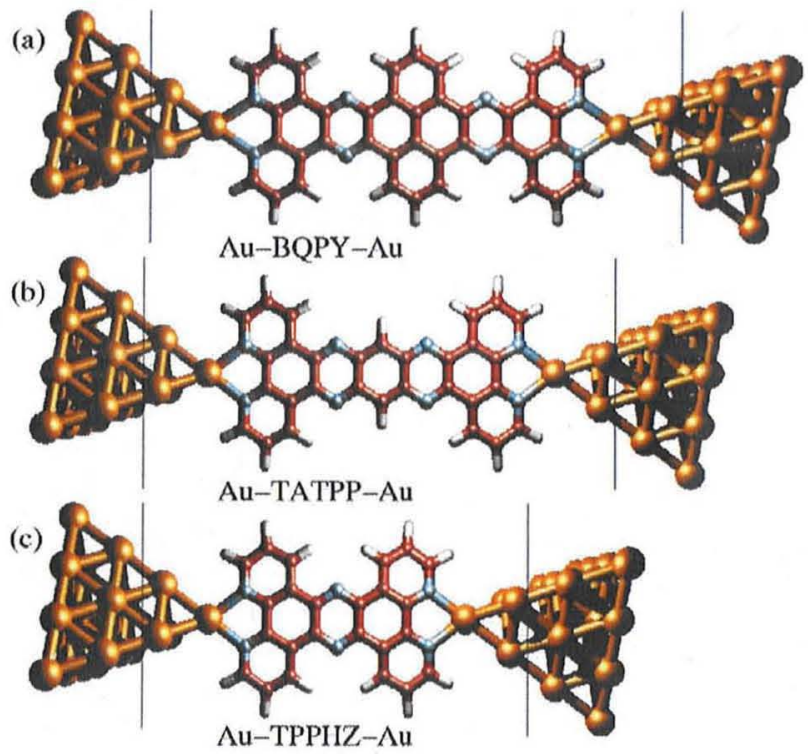

Figure 1. (Colour online) Geometries of the Au-BQPY-Au (a), $\mathrm{Au}-\mathrm{TATPP}-\mathrm{Au}(\mathrm{b})$, and Au-TPPHZ-Au (c) junctions. The black horizontal lines indicate where the junctions are cut into left and right electrode and extended-molecule. Yellow-gold atoms, blue — nitrogen, brown — carbon, and grey-hydrogen.

quinoxalino[2,3-i $i$ dipyrido[3,2- $\left.a: 2^{\prime}, 3^{\prime}-c\right]$ phenazine, commonly known as $9,11,20,22$-tetraazatetrapyrido[3,2- $a: 2^{\prime}, 3^{\prime}-c: 3^{\prime \prime}$, $\left.2^{\prime \prime}-l: 2^{\prime \prime \prime}, 3^{\prime \prime \prime}-n\right]$ pentacene (TATPP), and dipyrido $\left[3^{\prime \prime}, 2^{\prime \prime}: 5^{\prime}, 6^{\prime} ; 2^{\prime \prime \prime}\right.$, $\left.3^{\prime \prime \prime}: 7^{\prime}, 8^{\prime}\right]$ quinoxalino $\left[2^{\prime}, 3^{\prime}: 9,10\right]$ phenanthro[4,5-abc]dipyrido $\left[3,2-h: 2^{\prime}, 3^{\prime}-j\right]$ phenazine, often alternatively referred to as bis-\{dipyrido[3,2-f: $\left.2^{\prime}, 3^{\prime}-\mathrm{h}\right]$ quinoxalo $\}-\left[2,3-e: 2^{\prime}, 3^{\prime}-l\right]$ pyrene (BQPY). TATPP and BQPY can be considered as the parent molecule TPPHZ extended internally by one repeat unit of a fused quinoxaline group and a pyrazine-fused pyrene (phenanthro[4,5-fgh]quinoxaline) group, respectively.

DFT as implemented in the quantum chemistry package TURBOMOLE v5.7 [12] is used to determine the electronic and nuclear structures of these systems. The basis set is TURBOMOLE's standard Gaussian basis set, which is of split valence polarization quality for all non-hydrogen molecules [13] and the exchange correlation functional is
BP86 [14, 15]. We determine the geometries by placing the molecules symmetrically between two gold atoms and relaxing the structures whilst maintaining their symmetry. This minimalist treatment of the binding of the connecting gold atom probably underestimates the $\mathrm{Au}-\mathrm{N}$ bond length, an effect which would be partly cancelled by the overestimation of the bond length through the use of the generalized gradient approximation (GGA) [16]. The gold atoms are replaced after the relaxation process by gold fcc pyramids with a lattice parameter of $4.08 \AA$ oriented in the (111)-direction, where the pyramids consist of four layers, with $1,3,6$, and 10 atoms in the individual layers from the inside to the outside. In the case of BQPY, we relaxed the organic molecule without maintaining the symmetry after placing the pyramids. The resulting optimized geometries are depicted in figure 1 and their $\mathrm{Au}-\mathrm{N}$ bond lengths are given in table 1. The nitrogen atoms of the phenanthroline end-groups form bonds with the gold electrodes leading to biligation of the gold tip.

The conduction properties of these metal-molecule-metal junctions are ascertained using Green's function techniques and the Landauer formula expressed in a local, non-orthogonal basis, as discussed in detail in [18-20]. The junctions are therefore cut into three parts: the left $(\mathrm{L})$ and right $(\mathrm{R})$ electrode and the extended-molecule $(\mathrm{M})$, which contains the organic molecule and the inner two layers of each pyramid as indicated in figure 1 . The transmission channels are obtained, adiabatically tracked, and classified according to their conductance point group [21] as done in our previous work [22]. We also calculate the local density of states (LDOS) of the molecular orbitals (MO), details of which are provided in the supplementary data (available at stacks.iop.org/JPhysCM/20/295208).

TPPHZ is exemplified here in detail. The analysis of the other molecules can be found in the supplementary data (available at stacks.iop.org/JPhysCM/20/295208). While the geometric point group of TPPHZ is $\mathrm{D}_{2 \mathrm{~h}}$, after connection to the gold electrodes the atomic symmetry reduces to $\mathrm{C}_{2 \mathrm{~h}}$. As the Green's function formalism inherently removes all atomic symmetry operators that establish end-to-end symmetry in the system [21], the conductance point group controlling the symmetry of the conduction channels reduces to $\mathrm{C}_{\mathrm{s}}$, where the mirror plane is the expanse of the organic molecule. 


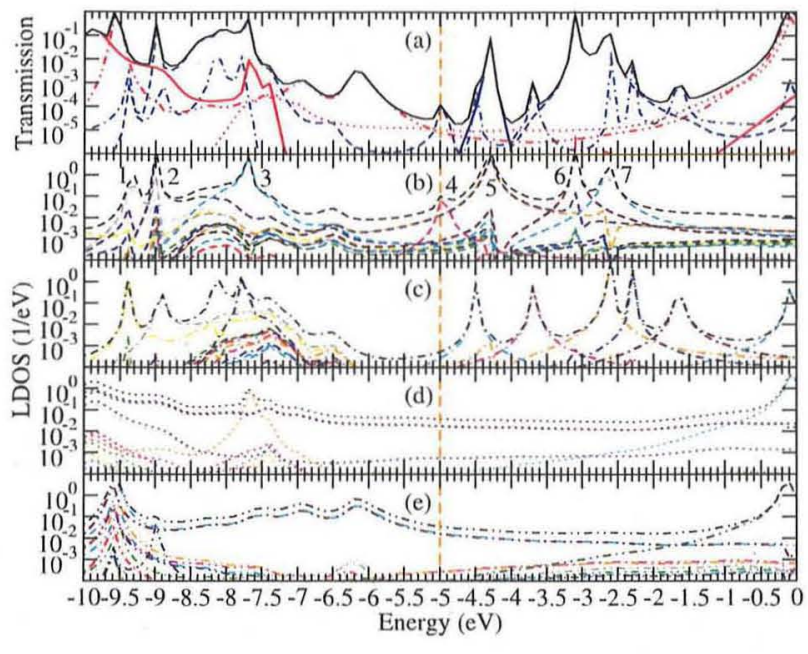

Figure 2. (Colour online) Transmission and LDOS versus energy for $\mathrm{Au}-\mathrm{TPPHZ}-\mathrm{Au}$. (a) Total transmission and transmission of the individual channels classified according to their conductance point group $\mathrm{C}_{\mathrm{s}}$. Black - total transmission, red-channels of $\mathrm{A}^{\prime}$ symmetry, blue - channels of $A^{\prime \prime}$ symmetry. The linestyle indicates the correspondence of the peaks' positions of that channel compared to the LDOS of TPPHZ's MOs classified in quasi- $\mathrm{C}_{2 v}$ symmetry (the geometric point group $\mathrm{D}_{2 \mathrm{~h}}$ of TPPHZ reduced by its end-to-end symmetry). The contributions of the individual MOs to the LDOS are given in different colours in panels (b)-(e). The black line in these panels corresponds to the sum of the contributions of MOs of that symmetry. (b) LDOS of MOs of $b_{1}$ symmetry. The orbitals with the largest contributions to the LDOS at the numbered peaks are shown in the supplementary data (available at stacks.iop.org/JPhysCM/20/295208). (c) LDOS of MOs of $a_{2}$ symmetry. (d) LDOS of MOs of $a_{1}$ symmetry. (e) LDOS of MOs of $b_{2}$ symmetry. The Fermi energy is given as the vertical, dashed, orange line.

Hence, the $\sigma$-orbitals of TPPHZ can only contribute to channels of $\mathrm{A}^{\prime}$ symmetry, while the $\pi$-orbitals can just take part in channels of $\mathrm{A}^{\prime \prime}$ symmetry. The transmission rigorously decomposed into $\mathrm{A}^{\prime}$ and $\mathrm{A}^{\prime \prime}$ channels is shown in figure 2(a). However, as the symmetry-breaking effects of the electrodes on the molecular orbitals are quite small, we sort the coupled MOs approximately according to the group $\mathrm{C}_{2 v}$ - the geometric point group $\mathrm{D}_{2 \mathrm{~h}}$ of the TPPHZ reduced only by its end-to-end symmetry [21], complying in fact with the conductance point group of the uncoupled molecule. Thus the MOs can be classified as being $\pi$ - or $\sigma$ orbitals as well as being roughly symmetric or antisymmetric with respect to the plane along the contact axis perpendicular to the expanse of the organic molecule. A description of the most significant molecular orbitals, the $\pi$-orbitals of $b_{1}$ symmetry, is provided in supplementary data (available at stacks.iop.org/JPhysCM/20/295208). The LDOS of the MOs sorted by their species are plotted in the individual panels (b)(e) in figure 2. We also show the sum of the LDOS for the MOs of each species. Comparing these sums with the transmission channels, we notice that they resemble each other very well. It is therefore possible to connect the individual transmission channels with a higher symmetry than the actual symmetry of conduction of the metal-molecule-metal junction, namely the conductance point group of the uncoupled molecule.

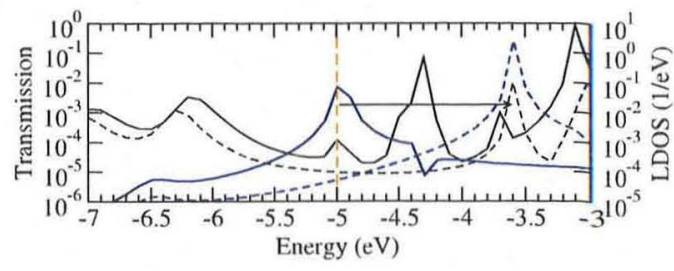

Figure 3. (Colour online) Effect of the scissors operator on the transmission and on the LDOS of the LUMO of Au-TPPHZ-Au (geometry (c) in figure 1). The transmission is shown in black and the LDOS of the LUMO in blue. The solid line corresponds to the uncorrected results and the dashed line to the empirically corrected ones. The Fermi energy of gold is given as the vertical, dashed, orange line.

Another striking feature of the transmission is that there is a peak at the Fermi energy. This peak is caused by the MO that has the shape of the LUMO of the free TPPHZ. However, the energy position of the orbital of the closed-shell TPPHZ molecule is not properly represented by DFT $[8,9]$, and its location at the Fermi energy leads to an overestimation of the tunnelling current. To account for this inadequacy, the positions of the occupied and virtual orbitals are corrected by calculating the ionization potential and electron affinity of the gold-TPPHZ-gold junction from the energy difference between the charge neutral system and the system with one electron added or respectively removed in the spirit of [10, 11]. We thus apply the scissors operator [11] and shift all of the occupied orbital energies by an amount chosen so that the HOMO energy corresponds to the ionization potential. Similarly, all of the vacant orbital energies are shifted by an amount chosen so that the LUMO energy corresponds to the electron affinity. Simple corrections of this form have been shown to be very effective in describing the ionization and electron-capture processes for molecules for which a wide range of experimental data is available $[8,9]$. Our method differs slightly from that of Quek et al [10, 11] and of Mowbray et al $[10,11]$ in that we do not assume that the free molecule is weakly coupled to ideal surfaces, but instead use explicit pyramidal electrodes. Therefore, we do not obtain our corrections from the free molecule together with an image charge model for ideal conducting surfaces, but rather calculate the differently charged states of the molecule coupled to the gold pyramids. Thus, charge reorganization in the metallic electrodes is treated intrinsically. A full description of the method is provided in supplementary data (available at stacks.iop.org/JPhysCM/20/295208).

A benchmark of the energy-level alignment method is given for 1,4-benzenediamine contacted by gold electrodes in summary in table 1 and in detail in supplementary data (available at stacks.iop.org/JPhysCM/20/295208), providing results in good agreement with the available experimental data [17] and other theoretical estimates [23, 24, 10, 11]. The effect of the empirical correction on the transmission through TPPHZ is shown in figure 3. The increase in the HOMOLUMO gap leads to a reduction of the uncorrected conductance of $1.2 \times 10^{-4} G_{0}$ to a corrected conductance of $1.0 \times 10^{-5} G_{0}$. Key properties of the calculated transmission are given in table 1. 


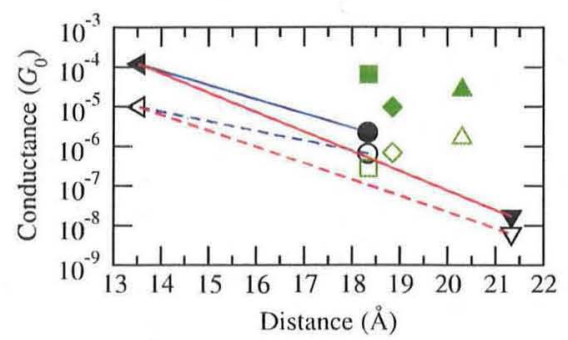

Figure 4. Conductances of the various molecules plotted as a function of the inter-electrode distance. Exponential fits to the data are shown, where the lines are fitted to Au-TPPHZ-Au and Au-TATPP-Au (blue) or Au-BQPY-Au (red). The solid lines correspond to the uncorrected data (filled symbols), while the dashed lines correspond to the empirically corrected data (open symbols). Left pointing triangle-Au-TPPHZ-Au, circle-Au-TATPP-Au, down pointing triangle-Au-BQPY-Au, squares-AuTATPP-1-linear-Au (figure 15(a) of the supplementary data (available at stacks.iop.org/JPhysCM/20/295208)), up pointing triangles-Au-TATPP-1-diagonal-Au (figure 15(b) of the supporting information (available at stacks.iop.org/JPhysCM/20/295208)), diamonds-Au-TATPP-2-Au (figure 15(c) of the supporting information (available at stacks.iop.org/JPhysCM/20/295208)).

We perform the same type of analysis for TATPP and BQPY and the results are given briefly in table 1 and in full in supplementary data (available at stacks.iop.org/JPhysCM/20/295208). The results for TATPP are qualitatively similar to the results of TPPHZ with a lower conductance as expected for the longer molecule. Due to the additional angularly fused rings of BQPY compared to the other two molecules, the LUMO of BQPY has a peculiar nodal structure of a very high energy [7], leading to a qualitatively different transmission around the Fermi energy. Further, BQPY presents transmission around the Fermi energy mainly through three different channels of which two can be attributed to $\sigma$-orbitals and one to a $\pi$-orbital according to the resemblance of the LDOS and the channel's transmission. The low contribution of $\pi$-orbitals to the conductance in extended $\pi$-conjugated molecules is a rare feature in single-molecule conduction and leads to a lower conductance than what would be expected from extrapolating the results for TPPHZ and TATPP. Figure 4 gives the conductances of all the investigated molecules as a function of the bridge length. An exponential fit to TPPHZ and TATPP gives an inverse decay length $(\beta)$ of approximately $0.8 \AA^{-1}$ for the raw conductances and $\beta$ equals $0.6 \AA^{-1}$ for the empirically corrected ones. These values, describing the conductance through linear fused quinoxaline groups, are quite large, of the order of the value of $0.73 \AA^{-1}$ evaluated by similar means for diaminoalkanes [22] and much higher than the value of $0.28 \AA^{-1}$ for dithiolated oligophenylene derivatives [25]. Simply having a $\pi$ conductor is thus insufficient to guarantee low inverse decay length. Further, the decay constants for adding pyrazine-fused pyrene groups to a linear chain, as obtained by comparing the conductances of TPPHZ and BQPY, are $1.1 \AA^{-1}$ (raw) and $0.9 \AA^{-1}$ (corrected), showing even poorer tunnelling.

Also, the low conductances calculated for TPPHZ indicate that 1,10-phenanthroline is poorly suited as a
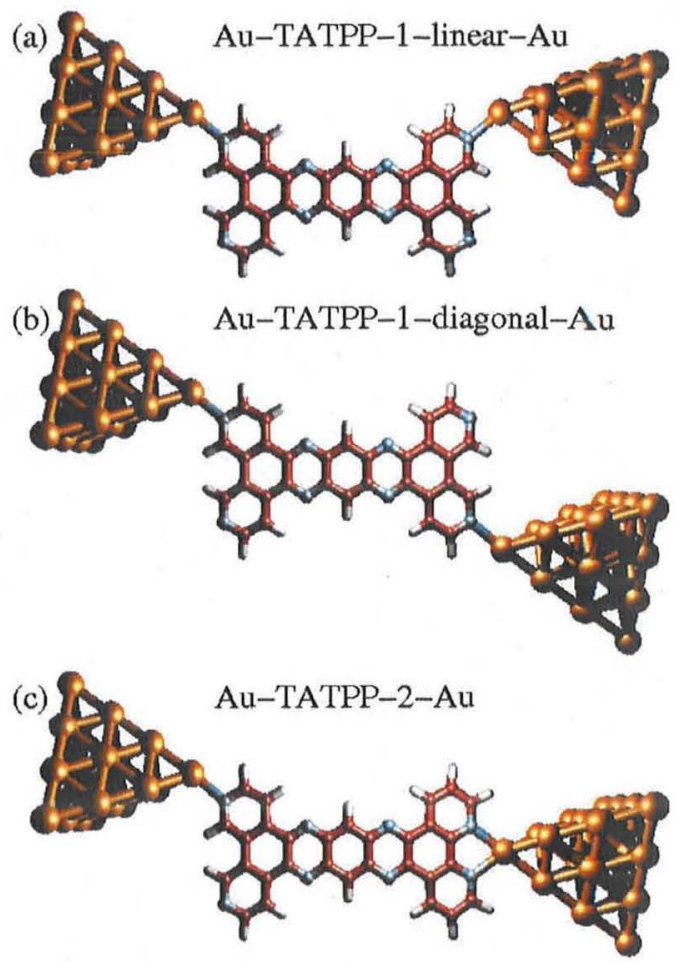

Figure 5. (Colour online) Variants of Au-TATPP-Au considered: (a) Au-TATPP-1-linear-Au, (b) Au-TATPP-1-diagonal-Au, and (c) Au-TATPP-2-Au. Yellow-gold atoms, blue-nitrogen, brown - carbon, and grey-hydrogen.

head group. Compared to related linkers such as amines and pyridines, 1,10-phenanthroline is a bidentate ligand and hence offers two paths for the current to flow from the gold into the molecule. To investigate the effect of this biligation on the conduction, derivatives of TATPP, dipyrido[ $\left[4^{\prime}, 3^{\prime}: 5,6 ; 3^{\prime \prime}, 4^{\prime \prime}: 7,8\right]$ quinoxalino[2,3$i]$ dipyrido $\left[4,3-a ; 3^{\prime}, 4^{\prime}-c\right]$ phenazine (TATPP-1) and dipyrido $\left[4^{\prime}, 3^{\prime}: 5,6 ; 3^{\prime \prime}, 4^{\prime \prime}: 7,8\right]$ quinoxalino[2,3-i]dipyrido $\left[3,2-a ; 2^{\prime}, 3^{\prime}-c\right]$ phenazine (TATPP-2) (see figure 5) are examined. We relaxed these organic molecules and placed the pyramids at the same position with respect to the binding nitrogen as in the case of TATPP. Structure TATPP-1 facilitates only monodentate binding, and two high-symmetry electrode connections, one 'linear' and one 'diagonal' with respect to the TATPP-1 coordination sites are considered; TATPP-2 is the intermediate species between these molecules and TATPP, facilitating bidentate binding at one end through its 1,10-phenanthroline unit but only monodentate binding at the other end.

The conductances of all of the TATPP-related junctions are listed in table 1 , along with their binding energies ( $\left.E_{\mathrm{BE}}\right)$ evaluated as $E_{\mathrm{BE}}=2 \times E_{\mathrm{Au} \text {-pyramid }}+E_{\mathrm{OM}}-E_{\mathrm{tot}}$, where $E_{\mathrm{Au}-\text { pyramid }}$ is the energy of the free gold pyramid, $E_{\mathrm{OM}}$ the energy of the isolated molecule, and $E_{\mathrm{tot}}$ the energy of the coupled metal-molecule-metal junction; further information is also available in supplementary data (available at stacks.iop.org/JPhysCM/20/295208). The binding energy decreases in steps of approximately $0.5 \mathrm{eV}$ when going from the bidentate over the mixed (one side bidentate, one side 
monodentate) to the monodentate structures. The binding energies of the monodentate structures are approximately $1.2 \mathrm{eV}$ ( $0.6 \mathrm{eV}$ per binding side), which are in the range expected from inspections of pyridine and 4, 4'-bipyridine binding to gold surfaces [26-28], and from the binding of amines to gold electrodes in the case of 1,4-benzenediamine (see table 1 and [24]). As expected, the use of the bidentate linker should thus strongly enhance the formation of the metal-organic interface. However, the raw conductances of the monodentate linker appears much higher than that of the bidentate one, with TATPP-2, TATPP-1-diagonal, and TATPP-1-linear being 4, 12, and 28 times larger than that for TATPP, respectively. These results arise as a manifestation of the change in the azine substitution pattern and any interference that the bidentate ligand may support. The corrected conductances do not show such a clear effect, however, with those for TATPP-2, TATPP-1-diagonal, and TATPP-1-linear being 1.0, 2.5, and 0.4 times that for TATPP, respectively. However, no correlation is found between the number of connections from the lead to the molecule at the gold-azine bottleneck and the overall conductance.

In summary, we see that use of extended $\pi$-conjugated molecules as molecular conductors does not provide an automatic route for the construction of high-conductivity longlength structurally sound molecular wires. While the 1-10phenanthroline linked fused molecules considered here are indeed structurally sound, the multiple conduction pathways present at the lead-molecule bottleneck do not always result in higher system conductivity. The results obtained are likely to apply more widely than just to the quinoxaline and pyrazinefused pyrene spacers considered herein but to many other synthetically feasible small-molecule graphene-like fragments as well.

Finally, we see that while DFT has significant problems in providing a realistic description of the orbital energies that control conduction in azine-linked systems, empirical corrections can be introduced that provide order-of-magnitude corrections. This allows useful a priori theoretical predictions of the conductance to be made so that chemically significant features can be examined.

\section{Acknowledgments}

SW and JRR thank The Australian Research Council for funding this research, as well as the Australian Partnership for Advanced Computing (APAC). FP acknowledges the funding of a Young Investigator Group at KIT.

\section{References}

[1] Tao N J 2006 Nat. Nanotechnol. 1 173-81

[2] Reimers J R, Hall L E, Crossley M J and Hush N S 1999 J. Phys. Chem. A 103 4385-97

[3] Li X L, Wang X R, Zhang L, Lee S W and Dai H J 2008 Science 319 1229-32

[4] Son Y W, Cohen M L and Louie S G 2006 Nature 444 347-9

[5] Katsnelson M I 2006 Mater: Today 10 20-7

[6] Geim A K and Novoselov K S 2007 Nat. Mater. 6 183-91

[7] Chiorboli C, Indelli M T and Scandola F 2005 Molecular Wires and Electronics (Topics in Current Chemistry) vol 257, ed L De Cola (Berlin: Springer) pp 63-102

[8] Reimers J R, Cai Z L, Bilic A and Hush N S 2003 Ann. New York Acad. Sci. 1006 235-51

[9] Bilic A, Reimers J R and Hush N S 2005 J. Chem. Phys. 122094708

[10] Quek S Y, Venkataraman L, Choi H J, Louie S G, Hybertsen M S and Neaton J B 2007 Nano Lett. 7 3477-82

[11] Mowbray D J, Jones G and Thygesen K S 2008 J. Chem. Phys. 128111103

[12] Ahlrichs R, Bär M, Häser M, Horn H and Kölmel C 1989 Chem. Phys. Lett. 162 165-9

[13] Schäfer A, Horn H and Ahlrichs R 1992 J. Chem. Phys. 97 2571-7

[14] Becke A D 1988 Phys. Rev. A 38 3098-100

[15] Perdew J P 1986 Phys. Rev. B 33 8822-4

[16] Lambropoulos N A, Reimers J R and Hush N S 2002 J. Chem. Phys. 116 10277-86

[17] Venkataraman L, Park Y S, Whalley A C, Nuckolls C, Hybertsen M S and Steigerwald M L 2007 Nano Lett. $7502-6$

[18] Pauly F 2007 Phase-coherent electron transport through metallic atomic-sized contacts and organic molecules Institut für Theoretische Festkörperphysik (Karlsruhe: Universität Karlsruhe (TH))

[19] Wohlthat S, Pauly F, Viljas J K, Cuevas J C and Schön G 2007 Phys. Rev. B 76075413

[20] Pauly F, Viljas J K, Cuevas J C and Schön G 2008 Phys. Rev. B 77155312

[21] Solomon G C, Gagliardi A, Pecchia A, Frauenheim T, Di Carlo A, Reimers J R and Hush N S 2006 J. Chem. Phys. 125184702

[22] Wohlthat S, Pauly F and Reimers J R 2008 Chem. Phys. Lett. 454 284-8

[23] Ning J, Li R, Shen X, Qian Z, Hou S, Rocha A R and Sanvito S 2007 Nanotechnology 18345203

[24] Li Z and Kosov D S 2007 Phys. Rev. B 76035415

[25] Viljas J K, Pauly F and Cuevas J C 2007 Phys. Rev. B 76033403

[26] Hou S M, Ning J, Shen Z Y, Zhao X Y and Xue Z Q 2006 Chem. Phys. 327 1-9

[27] Bilic A, Reimers J R and Hush N S 2002 J. Phys. Chem. B 106 6740-7

[28] Stadler R, Thygesen K S and Jacobsen K W 2005 Phys. Rev. B 72 241401(R) 